\title{
Aplicación de la metodología aprendizaje servicio en un curso de Química
}

\section{Rodrigo del Río}

Departamento de Química Inorgánica, Facultad de Química, Pontificia Universidad Católica de Chile, Santiago, Chile.

Centro de Investigación en Nanotecnología y Materiales Avanzados (CIEN-UC).

\section{Resumen}

Se presenta la experiencia desarrollada en el curso "Química en la naturaleza y la sociedad" aplicando la metodología aprendizaje servicio. Éste es un curso de primer año de la carrera de Química de la Pontificia Universidad Católica de Chile. El servicio prestado consiste en desarrollar actividades didácticas para niños de cuarto año de enseñanza primaria en establecimientos de alta vulnerabilidad, en conjunto con el Centro Científico de la Casa de la Cultura del municipio de La Pintana. La aplicación de la metodología permitió trabajar el desarrollo de habilidades genéricas en los estudiantes de química, tales como el trabajo en equipo y el compromiso social y a la vez conseguir en los estudiantes aprendizajes significativos referentes a los objetivos asociados a la asignatura. La evaluación de la actividad por parte de los alumnos indica que ellos sienten que aprenden mejor los contenidos y a la vez que perciben que desarrollan sus habilidades genéricas.

\section{Palabras clave}

Química, Educación Primaria, habilidades genéricas.

Fecha de recepción: 29/IX/2015

Fecha de aceptación: 29/X/2015 


\section{Application of service learning methodology in a course of Chemistry}

\section{Abstract}

The paper describe the experience of the course "Chemistry in nature and society" applying the service-learning methodology. This is a first year course included in the chemistry academic program at the Pontificia Universidad Católica de Chile. The service provided is a set of educational activities for children under four course of primary education in one of the most vulnerable communities in the metropolitan region of Santiago. The experience has been useful to focus on the development of generic skills in students, such as teamwork and social consciousness. The student's evaluation of the activity indicates that they are motivated to learn deeply the course contents and perceive that generic skills were enhanced by the activity.

\section{Keywords}

Chemistry, primary education, generic skills. 


\section{Introducción}

Como es sabido aprendizaje servicio $(A+S)$ es una metodología pedagógica que relaciona los contenidos de un curso con un servicio a la comunidad. Con esta metodología se genera una sociedad entre la comunidad y los estudiantes. Mediante la actividad realizada y las reflexiones adecuadas, es posible conseguir que la comunidad reciba un servicio de calidad y que los alumnos tengan aprendizajes significativos $y$ formación valórica (Jouannet et al, 2013).

$A+S$ es aplicada en una infinidad de asignaturas de diferentes carreras universitarias e incluso a nivel de educación secundaria, pero son escasos los casos en el área científica. En particular, en carreras del área química, existen pocos artículos que mencionen como se aplica $A+S$ y esto es debido a que la Química es considerada como una carrera solitaria desde la perspectiva de la relación con la comunidad, ya que el profesional se desempeña principalmente al interior de un laboratorio. Además se debe considerar que la percepción social de la Química no es buena debido a que se la relaciona con la contaminación ambiental (Mammino, 2001). La Sociedad Americana de Química ha considerado que se debe estimular el uso de $A+S$ en los departamentos de Química principalmente en las áreas de química ambiental y en apoyo a la labores educacionales en nivel primario y secundario (Wiegand $y$ Strait, 2000).

En la Pontificia Universidad Católica de Chile se ha implementado esta metodología desde el año 2004 y actualmente se cuenta con más de un 
centenar de cursos que la utilizan (Jouannet et al, 2013). En la Facultad de Química, se imparte el curso "Química en la Naturaleza y la Sociedad" perteneciente al primer año del programa académico de Química.

\section{Diseño y descripción de la actividad}

El curso de primer año "Química en la Naturaleza y la Sociedad", pretende que los alumnos sean capaces de relacionar fenómenos químicos con conceptos básicos de la disciplina, tales como estructura molecular y reactividad. Dentro de los objetivos del curso se tiene fomentar una actitud crítica en el manejo de información científica, expresar sus conocimientos verbalmente utilizando el lenguaje científico adecuado y estimular el trabajo en equipo. Los contenidos abarcan diferentes fenómenos químicos, tales como, disminución de la capa de ozono, calentamiento global, contaminación atmosférica y reciclaje de materiales.

En este contexto se comenzó a utilizar $A+S$ en 2011, lo que implicó tomar contacto con el municipio de La Pintana -uno de los municipios más vulnerables de la región metropolitana de Santiagoparticularmente con su Centro Científico, y se diseñó en conjunto la actividad $\mathrm{A}+\mathrm{S}$. Este proyecto comienza con la conformación de equipos de trabajo de cuatro estudiantes, heterogéneos en cuanto a sexo, origen regional y educacional. A estos grupos se les asignó un tema que está incluido en los contenidos de Ia asignatura, previamente consensuados con el socio comunitario. Los estudiantes elaboraron un trabajo de investigación para estimular el 
aprendizaje significativo del tema y a la vez diseñaron una actividad didáctica del mismo. Esta actividad es ejecutada con grupos de cuatro niños de cuarto año de educación primaria de colegios públicos del municipio en tres sesiones de 90 minutos realizadas con los niños en dependencias del municipio.

La evaluación de esta actividad involucra el $40 \%$ de la calificación final del curso y se divide en evaluación del trabajo de investigación, de la actividad realizada, evaluación de pares y autoevaluación con rúbricas adecuadas que cubren criterios referidos a la profundidad de los contenidos, redacción y ortografía, pertinencia de la actividad, responsabilidad, participación y capacidad de trabajar en equipo.

\section{Instancias de reflexión}

Como es sabido la reflexión es una dimensión crucial de la metodología $A+S$ (Eyler, 2001) y para este curso se ha diseñado una reflexión inicial en la que se discute como la actividad requiere del aprendizaje de los contenidos involucrados en el tema y como esto va de la mano con la responsabilidad que tienen los alumnos al traspasar información científica a los niños que participan de la actividad. Para esto, se les solicita opinar frente a frases tales como: "si conozco muy bien un tema, soy capaz de explicárselo a un niño" o "debo aprender más para responderle al niño que para aprobar un examen". Existe una segunda reflexión que apunta a la formación valórica asociada a la actividad; en esta se les pregunta a los alumnos su opinión acerca de quiénes son las personas 
que los niños admiran, sus modelos a seguir y que los ven en los medios de comunicación (futbolistas, artistas, etc) y se motiva a los estudiantes a que deben preocuparse de enseñar y ser modelo de persona y mostrarles a los niños que estudiar para ser profesional, puede ser muy valioso para su formación. En la última reflexión evaluamos en conjunto el grado de éxito alcanzado y la posibilidad de mejorar las actividades.

\section{Evaluación de la actividad}

Mediante encuestas aplicadas al finalizar el curso, los estudiantes indicaron, con mas de $80 \%$ de acuerdo, que la actividad fue importante en su formación. Cualitativamente, se puede destacar la opinión de un alumno que mencionó: "aprendí aparte del tema que nos tocó, a trabajar en equipo, a sociabilizar, a compartir ideas, enfrentar problemas y buscar soluciones". A partir de ésta y de otras opiniones, se puede inferir que se logró un aprendizaje significativo del tema y se estimularon habilidades genéricas. Otro alumno planteó: "La actividad me dejo aprendizajes técnicos, todo lo que llevo consigo el trabajo, informes, presentaciones, una nueva visión de la ciencia, pero también aprendizaje humano en el sentido que nos dimos cuenta de la realidad de esos niños que viven en una de las comunas mas pobres de Santiago, como viven, la realidad de sus padres, su situación en el colegio, lo que me ayudó a valorizar mas la realidad que vivo, también que en conjunto hemos podido lograr ciertas metas, que en comparación a los niños cuyo núcleo familiar no estaba completo y se encuentran muy vulnerables" a partir de la cual se pudo constatar que se consiguió la 


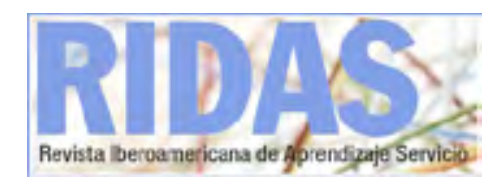

formación en valores que se persigue con la metodología. El socio comunitario ha decido continuar su trabajo con nosotros ya que considera que el servicio es adecuado. Así, las evidencias indican que los tres pilares del modelo $A+S$ UC están presentes en el curso: aprendizaje significativo, servicio de calidad y formación en valores.

En resumen, esta metodología permite desarrollar en los estudiantes aprendizajes más profundos de los contenidos, transferirles la responsabilidad de su autoaprendizaje, fortalecer la formación valórica de ellos y del profesor; ya que por ser el responsable del éxito de la actividad debe involucrarse con las personas que prestan el servicio y las que lo reciben, los niños. Así, se esta consiguiendo estimular habilidades genéricas más eficientemente que sin el uso de $A+S$, desde un curso de primer año y en una carrera científica.

\section{Conclusiones}

Considerando los pocos casos de aplicación de $A+S$ en cursos de carreras científicas, se puede concluir que en este caso particular, la metodología ha permitido obtener aprendizajes significativos en los estudiantes y a la vez promover el desarrollo de habilidades genéricas (trabajo en equipo, compromiso social, liderazgo) en ellos y también en el profesor responsable por hacerle participe de la realidad a la que enfrenta a los estudiantes.

\section{Referencias bibliográficas}

Eyler, J., (2001) Creating your reflection map. New directions for higher education, 114. 
Jouannet, C.; Salas, M.; Contreras, M. (2013) Modelo de implementación de Aprendizaje Servicio $(A+S)$ en la UC. Una experiencia que impacta positivamente en la formación profesional integral. Calidad en Educación. 39, 197-212.
Mammino, L. (2001) Algunas reflexiones sobre la imagen de la Química. Anales de la Real Sociedad Española de Química. 48-52.

Wiegand, D.; Strait, M. (2000) What is Service Learning? Journal of Chemical Education. 77, 1538-1539. 